Wei-Hong Yan* and Wan-Yao Chen

\title{
Crystal structure of $1,1^{\prime}$-(1,2-ethanediyl) bis(pyridin-1-ium) bis(1,2-dicyanoethene- 1,2-dithiolato-k ${ }^{2}$ S:S)nickel(II), $\mathrm{C}_{20} \mathrm{H}_{14} \mathrm{~N}_{6} \mathrm{NiS}_{4}$
}
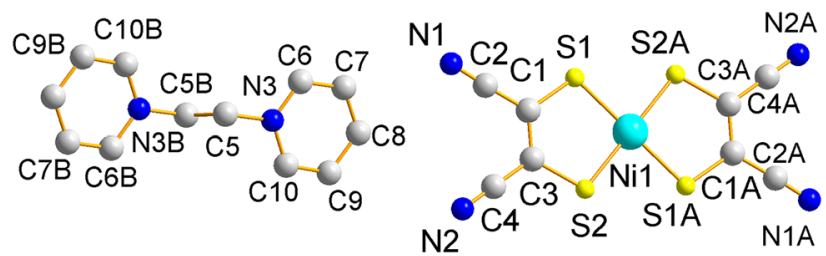

https://doi.org/10.1515/ncrs-2021-0433

Received November 8, 2021; accepted January 13, 2022;

published online January 31, 2022

\section{Abstract}

$\mathrm{C}_{20} \mathrm{H}_{14} \mathrm{~N}_{6} \mathrm{NiS}_{4}$, triclinic, $P \overline{1}$ (no. 2), $a=8.519(3) \AA$, $b=8.5956(16) \AA, c=8.6531(17) \AA, \alpha=116.404(3)^{\circ}$, $\beta=94.574(4)^{\circ}, \gamma=103.031(4)^{\circ}, V=541.0(2) \AA^{3}, Z=1$, $R_{g t}(F)=0.0445, w R_{\text {ref }}\left(F^{2}\right)=0.1147, T=296.15 \mathrm{~K}$.

CCDC no.: 2141877

Table 1 contains crystallographic data and Table 2 contains the list of the atoms including atomic coordinates and displacement parameters.

\section{Source of material}

All reagents and chemicals were purchased from commercial sources and used without further purification. The starting materials disodium maleonitriledithiolate and 1,1'(1,2-ethanediyl)bis(pyridin-1-ium)bromide were synthesized following the literature procedures [3, 4]. An aqueous solution (10 mL) of 1,1'-(1,2-ethanediyl)bis(pyridin-1-ium) bromide $(0.068 \mathrm{~g}, 0.2 \mathrm{mmol})$ was added slowly to an aqueous solution $(20 \mathrm{~mL})$ of disodium maleonitriledithiolate $(0.074 \mathrm{~g}, 0.4 \mathrm{mmol})$ and $\mathrm{NiCl}_{2} \cdot 6 \mathrm{H}_{2} \mathrm{O}(0.048 \mathrm{~g}$, $0.2 \mathrm{mmol}$ ), and the mixture was stirred at room temperature

\footnotetext{
*Corresponding author: Wei-Hong Yan, School of Biology and Chemical Engineering, Nanyang Institute of Technology, Nanyang 473004, China, E-mail: yyu_yu@163.com. https://orcid.org/0000-0002-6235-4061

Wan-Yao Chen, School of Biology and Chemical Engineering, Nanyang Institute of Technology, Nanyang 473004, China
}

Table 1: Data collection and handling.

\begin{tabular}{ll}
\hline Crystal: & Red block \\
Size: & $0.25 \times 0.21 \times 0.17 \mathrm{~mm}$ \\
Wavelength: & Mo $K \alpha$ radiation $(0.71073 \AA$ A $)$ \\
$\mu:$ & $1.30 \mathrm{~mm}^{-1}$ \\
Diffractometer, scan mode: & $\varphi$ and $\omega$ \\
$\theta_{\text {max }}$, completeness: & $28.5^{\circ}, 99 \%$ \\
$N(h k l)_{\text {measured }}, N(h k l)_{\text {unique }}, R_{\text {int }}:$ & $3974,2708,0.027$ \\
Criterion for $I_{\text {obs }}, N(h k l)_{\text {gt }}:$ & $I_{\text {obs }}>2 \sigma\left(I_{\text {obs }}\right), 2157$ \\
$N(\text { param })_{\text {refined }}:$ & 142 \\
Programs: & SHELX [1], Bruker [2]
\end{tabular}

for several minutes. A red precipitate was filtered off, washed by water and dried under vacuum. The precipitate was dissolved in DMF with ether diffusion. Two weeks later yielded red crystals.

\section{Experimental details}

Absorption corrections were applied by using multi-scan program. The structure was refined based on $F^{2}$ with the SHELXL software package. Hydrogen atoms were treated as riding atoms. The $U_{\text {iso }}$ values of the hydrogen atoms were set to $1.2 U_{\text {eq }}(\mathrm{C})$.

\section{Comment}

The development of polythiene complexes have captured much attention because of their variable structures and properties on molecular conductors, magnets, catalysts, and nonlinear optical, etc. [5-9]. The maleonitriledithiolate (mnt) ligand is one of the most popular ligands in this area. It is crucial to design and prepare suitable multifunctional salts to achieve new molecular arrangements, resulting in developing unique stacking schemes of $\left[\mathrm{M}(\mathrm{mnt})_{2}\right]^{2-}$ anions and properties. Recently, our effort have been devoted to maleonitriledithiolate complexes using different cations [10-12].

The title complex crystallizes in triclinic $P \overline{1}$ space group, with one half of $\left[\mathrm{Ni}(\mathrm{mnt})_{2}\right]^{2-}$ dianion and one half of 
Table 2: Fractional atomic coordinates and isotropic or equivalent isotropic displacement parameters $\left(\AA^{2}\right)$.

\begin{tabular}{lrrrr}
\hline Atom & $\boldsymbol{x}$ & $\boldsymbol{y}$ & $\boldsymbol{z}$ & $\boldsymbol{U}_{\text {iso }} / \boldsymbol{U}_{\text {eq }}$ \\
\hline C1 & $0.2296(4)$ & $0.3660(4)$ & $0.5637(4)$ & $0.0185(6)$ \\
C2 & $0.2905(4)$ & $0.5305(4)$ & $0.5563(4)$ & $0.0204(7)$ \\
C3 & $0.3197(4)$ & $0.3216(4)$ & $0.6662(4)$ & $0.0175(6)$ \\
C4 & $0.4793(4)$ & $0.4371(4)$ & $0.7693(4)$ & $0.0194(7)$ \\
C5 & $1.0133(4)$ & $1.4167(4)$ & $0.9267(4)$ & $0.0187(7)$ \\
H5A & 1.006242 & 1.425756 & 0.818658 & $0.022^{*}$ \\
H5B & 1.121933 & 1.408168 & 0.957223 & $0.022^{*}$ \\
C6 & $0.7543(4)$ & $1.1766(4)$ & $0.7662(4)$ & $0.0178(6)$ \\
H6 & 0.744419 & 1.223846 & 0.689105 & $0.021^{*}$ \\
C7 & $0.6339(4)$ & $1.0267(4)$ & $0.7451(4)$ & $0.0225(7)$ \\
H7 & 0.541736 & 0.973156 & 0.653955 & $0.027^{*}$ \\
C8 & $0.6501(5)$ & $0.9569(5)$ & $0.8581(5)$ & $0.0303(8)$ \\
H8 & 0.569174 & 0.856050 & 0.843959 & $0.036^{*}$ \\
C9 & $0.7887(5)$ & $1.0377(5)$ & $0.9949(5)$ & $0.0359(10)$ \\
H9 & 0.801802 & 0.991621 & 1.072584 & $0.043^{*}$ \\
C10 & $0.9051(5)$ & $1.1868(5)$ & $1.0116(5)$ & $0.0302(8)$ \\
H10 & 0.998230 & 1.242254 & 1.101628 & $0.036^{*}$ \\
N1 & $0.3333(4)$ & $0.6627(4)$ & $0.5477(4)$ & $0.0259(6)$ \\
N2 & $0.6084(4)$ & $0.5299(4)$ & $0.8526(4)$ & $0.0288(7)$ \\
N3 & $0.8864(3)$ & $1.2536(4)$ & $0.9002(3)$ & $0.0179(6)$ \\
Ni1 & 0.000000 & 0.000000 & 0.500000 & $0.01449(16)$ \\
S1 & $0.03407(10)$ & $0.22651(11)$ & $0.44255(10)$ & $0.01830(19)$ \\
S2 & $0.24209(10)$ & $0.11917(11)$ & $0.67378(10)$ & $0.01782(19)$ \\
\hline & & & &
\end{tabular}

1,1'-(1,2-ethanediyl)bis(pyridin-1-ium) dication in the asymmetric unit (see the Figure). Each $\mathrm{Ni}(\mathrm{II})$ ion is coordinated by four sulfur atoms, and exhibits a quasi-square planar coordination geometry. The Ni1-S bond lengths and S-Ni1-S bond angles are in 2.1787(10)-2.1798(9) $\AA$ and $88.12(3)-180.0^{\circ}$, respectively. These parameters are comparable to the reported $\left[\mathrm{Ni}(\mathrm{mnt})_{2}\right]^{2-}$ complexes [11]. The anions and cations form segregated columns. The $\mathrm{C}-\mathrm{H} \cdots \mathrm{N}$ interactions are observed between anions and cations [13]. The three-dimensional supramolecular structure is formed by such weak interactions.

Author contributions: All the authors have accepted responsibility for the entire content of this submitted manuscript and approved submission.

Research funding: This study was financially supported by the National Natural Science Foundation of China (21776063, U1704127).
Conflict of interest statement: The authors declare no conflicts of interest regarding this article.

\section{References}

1. Sheldrick G. M. Crystal structure refinement with SHELXL. Acta Crystallogr. 2015, C71, 3-8.

2. Bruker. SMART APEX-II CCD; Bruker AXS Inc.: Madison, WI, USA, 2006.

3. Simmons H. E., Blomstrom D. C., Vest R. D. Thiacyanocarbons. II. chemistry of disodium dimercaptomaleonitrile. J. Am. Chem. Soc. 1962, 84, 4756-4771.

4. Musilek K., Komloova M., Zavadova V., Holas O., rabinova M., Pohanka M., Dohnal V., Nachon F., Dolezal M., Kuca K., Jung Y. S. Preparation and in vitro screening of symmetrical bispyridinium cholinesterase inhibitors bearing different connecting linkageinitial study for Myasthenia gravis implications. Bioorg. Med. Chem. Lett 2010, 20, 1763-1766.

5. Coomber A. T., Beljonne D., Friend R. H., Brédas J. L., Charlton A., Robertson N., Underbill A. E., Kurmoo M., Day P. Intermolecular interactions in the molecular ferromagnetic $\mathrm{NH}_{4} \mathrm{Ni}(\mathrm{mnt})_{2} \cdot \mathrm{H}_{2} \mathrm{O}$. Nature 1996, 380, 144-146.

6. Robertson N., Cronin L. Metal bis-1,2-dithiolene complexes in conducting or magnetic crystalline assemblies. Coord. Chem. Rev. 2002, 227, 93-127.

7. Duan H. B., Ren X. M., Meng Q. J. One-dimensional (1D) $\left[\mathrm{Ni}(\mathrm{mnt})_{2}\right]^{-}$based spin-Peierls-like complexes: structural, magnetic and transition properties. Coord. Chem. Rev. 2010, 254, 1509-1522.

8. Wang P. Crystal structure of $\mathrm{Mn}(\mathrm{II})\left(\mathrm{H}_{2} \mathrm{O}\right)(2,13$-dimethyl3,6,9,12,18-pentaazabicyclo[12.3.1]octadeca1(18),2,12,14,16-pentaene) bis (maleonitriledithiolate)nickel(II), $\mathrm{C}_{23} \mathrm{H}_{25} \mathrm{MnN}_{9} \mathrm{NiOS}_{4}$. Z. Kristallogr. N. Cryst. Struct. 2012, 227, 511-512.

9. Perochon R., Barriere F., Jeannin O., Piekara-Sady L., Fourmigue $M$. A radical mixed-ligand gold bis(dithiolene) complex. Chem. Commun. 2021, 57, 1615-1618.

10. Yan W. H., Ji E. Y., Shen M. L., Li Z. Y., Li X., Xu X. L. Two ion-pair complexes constructed by $\left[\mathrm{M}(\mathrm{mnt})_{2}\right]^{n-}(\mathrm{M}=\mathrm{Ni}, \mathrm{Co}, \mathrm{mnt}=$ maleonitriledithiolate): syntheses, characterization and thermal stability. Chin. J. Struct. Chem. 2015, 34, 306-312.

11. Yan W. H., Pan H. Y. Crystal structure of 1,4-bis(methylpyridinium benzene) bis(1,2-dicyanoethene-1,2-dithiolato- $\left.\kappa^{2} S: S\right)$ nickel(II), $\mathrm{C}_{26} \mathrm{H}_{18} \mathrm{~N}_{6} \mathrm{NiS}_{4}$. Z. Kristallogr. N. Cryst. Struct. 2021, 236, 107-108.

12. Yan W. H., Liu X. G., Shen M. L., Pan H. Y. Crystal structure of $1,1^{\prime}$ (1,4-phenylenebis(methylene))bis(pyridin-1-ium) bis (1,2-dicyanoethene-1,2-dithiolato- ${ }^{2} S: S$ ) zinc(II), $\mathrm{C}_{26} \mathrm{H}_{18} \mathrm{~N}_{6} \mathrm{ZnS}_{4}$. Z. Kristallogr. N. Cryst. Struct. 2021, 236, 533-535.

13. Jeffrey G. A. Hydrogen-bonding: an update. Crystallogr. Rev. 2003, 9, 135-176. 